\title{
AGN spiral galaxies in groups: effects of bars
}

\author{
Sol Alonso ${ }^{1,2}$, Georgina Coldwell ${ }^{1,2}$, and Diego G. Lambas ${ }^{1,2,3}$ \\ ${ }^{1}$ Consejo Nacional de Investigaciones Científicas y Técnicas, CONICET, Argentina \\ e-mail: solalonsog@gmail.com \\ 2 FCEFyN, Universidad Nacional de San Juan, 5400 San Juan, Argentina \\ 3 IATE, OAC, Universidad Nacional de Córdoba, Laprida 854, X5000BGR Córdoba, Argentina
}

Received 3 July 2014 / Accepted 11 September 2014

\begin{abstract}
Aims. We explore properties of barred active spiral galaxies in groups and clusters selected from the Sloan Digital Sky Survey data release 7 (SDSS-DR7), with the aim of assessing the effects of bars on active galactic nuclei (AGN) and the role of the high density environment.

Methods. We identified barred active galaxies that reside in groups and clusters by cross-correlating the total barred AGN sample with the SDSS-DR7 group catalog. With the goal of providing a suitable quantification of the effects of bars, a reliable control sample of unbarred active galaxies in high density environments with similar redshift, magnitude, morphology, and bulge size distributions was also constructed.

Results. We found that the fraction of barred AGN galaxies in groups and clusters $(\approx 38 \%)$ is higher than those in the total barred AGN sample $(\approx 28 \%)$, indicating that AGN spiral galaxies in groups are more likely to be barred than those in the field. We also found that barred AGN galaxies are more concentrated towards the group centers than the other unbarred AGN group members. In addition, barred AGN host galaxies show an excess of population dominated by red colors, with respect to the control sample, suggesting that bars produce an important effect on galaxy colors of AGN hosts. The groups of AGN galaxies with and without bars show similar virial masses; however, the host groups of the barred AGN exhibit a larger fraction of red colors than the host groups of the corresponding unbarred active galaxies in the control sample. Color-magnitude relations of both host groups of AGN differ significantly: the host group colors of barred active galaxies display distributions spreading toward red populations, at the same $\left(M_{r}\right)_{\text {Group }}$, with respect to the host groups of the unbarred AGN objects. This trend is more significant in less massive groups than in groups with $M_{\text {Virial }}>10^{13.5} M_{\odot}$. Barred active galaxies show an excess of nuclear activity compared to galaxies without bars in the control sample. We found that barred active galaxies located farther from the group-center have stronger Lum[OIII], while the nuclear activity in AGN galaxies without bars remain approximately constant with the group-centric distance. In addition, for both AGN samples, nuclear activity increases in bluer host groups however, barred active objects systematically show higher Lum[OIII] values, irrespective of the global group colors. Our findings suggest that the efficiency of bars to transport material towards the more central regions of the AGN galaxies in high density environments reveals an important dependence on the localization of objects within the group/cluster and on the host group colors.
\end{abstract}

Key words. galaxies: active - galaxies: spiral - galaxies: groups: general - galaxies: formation

\section{Introduction}

Galactic bars are believed to be directly related to the dynamical evolution of their host spiral galaxies. Several studies of numerical simulation show that bars can efficiently transport gas from the outer regions of galaxies to the central kiloparsec scale (Weinberg 1985; Debattista \& Sellwood 1998; Athanassoula 2003). The gas clouds suffer shocks by interaction with the edges of the bars, producing angular momentum losses and allowing a flow of gas toward the innermost central regions of the barred galaxies (Shlosman et al. 1990). Given the high efficiency of gas inflow, the presence of bars plays an important role in enhancing the nuclear star formation (Heckman 1980; Hawarden et al. 1986; Devereux 1987; Arsenault 1989; Huang et al. 1996; Ho et al. 1997; Martinet \& Friedli 1997; Emsellem et al. 2001; Knapen et al. 2002; Jogee et al. 2005; Hunt et al. 2008).

In addition, the bar-driven material toward the most inner regions has been considered an efficient mechanism for triggering activity in active galactic nuclei (AGN; Corsini et al. 2003; Combes et al. 1993). Shlosman et al. (1989) proposed a model that they called "bars within bar" as a mechanism for fueling AGN galaxies. In this scenario, the external bars transport gas on a scale of a few parsec and the material is accumulated. It shows a gravitational instability producing a secondary bar, which allows gas to approach closer to a central black hole (Mulchaey \& Regan 1997; Maciejewski \& Sparke 1997; Ho et al. 1997). Different observational studies, using the Hubble Space Telescope images and the spectroscopic data obtained from ISAAC/VLT and SAURON (Emsellem et al. 2001; Malkan et al. 1998; Laine et al. 2002; Carollo et al. 2002) have confirmed the presence of secondary bars, by observing nuclear bars inside large-scale external bars.

There is still a debate on the observational analysis that shows different results regarding the effects produced by bars on the central nuclear activity. For instance, Ho et al. (1997) found that bars have no significant effect on the AGN activity in a sample of 187 barred spiral galaxies. Lee et al. (2012) also used a sample of AGN, selected with the Kewley et al. (2001) classification, from the Sloan Digital Sky Survey data release 7 (SDSSDR7) volume limited sample of late-type galaxies $(b / a>0.7)$ concluding that bars do not have an important effect on the nuclear activity. However, Oh et al. (2012) found evidence that bars 
enhance central activity in blue galaxies with low black hole masses from a sample of late-type active galaxies $(b / a>0.6)$ selected from SDSS-DR7 with the Kauffmann et al. (2003) criteria. In the analysis, Lee et al. (2012) do not include the composite galaxies within the sample of AGN host and because these galaxies are in general, younger and less massive (Kewley et al. 2006) than the AGN sample defined by the Kewley et al. (2001) criteria. So, the different AGN selection criteria could affect the results related to the effect of enhanced AGN activity by bars.

More recently, Alonso et al. (2013, hereafter A13) studied a sample of face-on barred AGN spiral galaxies, in comparison with a suitable control sample of unbarred active galaxies with similar redshift, magnitude, morphology, bulge sizes, and local environment distributions. They found that barred AGN galaxies systematically show a higher fraction of powerful activity with respect to unbarred AGN in the control sample. In addition, the analysis of the accretion rate onto the central black holes shows that barred AGN host galaxies show an excess of objects with high accretion rate values with respect to unbarred active ones.

Galaxy properties (star formation, gas content, morphology, etc.) show a strong dependence on the local environment in which galaxies reside. Therefore, to have a better understanding of the formation and evolution of bars, an important point is to analyze, not only the internal structures of spiral galaxies, but also the external influence on their host environment. Nevertheless, the dependence between the bar fraction and environment is a controversial point since different studies show contradictory results. Several observational works found that the bar galaxy fraction does not depend on the environment (van den Bergh 2002; Mendez-Abreu et al. 2010; Martínez \& Muriel 2011; Lee et al. 2012). However, Elmegreen et al. (1990) found a higher bar fraction in pair systems for a sample of spiral galaxies. In addition, Eskridge et al. (2000) show that the bar fraction in the Fornax and Virgo clusters is slightly higher than the average value for fields. Moreover, from the analysis of galaxies in the Shapley-Ames Catalog, van den Bergh (2002) found that the fraction of barred galaxies in cluster environments is larger than in lower density regions. Recently, Skibba et al. (2012) show that barred galaxies tend to reside in denser environments than their unbarred counterparts for a large sample obtained from the Galaxy Zoo 2 project (Lintott et al. 2011), finding a clear environmental dependence on barred galaxies.

Motivated by these finds, here we analyze the effect of bars on active nuclei galaxies and the role played by high density environments. We study active galaxies with and without bars within groups and clusters with the aim of assessing whether high density environments play a significant role in modifying the host properties and nuclear activity of barred active galaxies. For this purpose, using data from the SDSS-DR7, we obtain samples of barred and unbarred AGN residing in groups and clusters of galaxies. We analyze the spatial distribution, host galaxy properties, and nuclear activity of barred AGN in high density regions, and compare them to those of unbarred active galaxies from a suitable control sample, with the aim of obtaining additional clues about the interplay between the high density environments and barred AGN galaxies.

This paper is structured as follows. Section 2 describes the procedure used to construct the catalog of barred AGN group galaxies from SDSS-DR7, and the control sample selection criteria. In Sect. 3, we study the barred and unbarred AGN relative position in groups/clusters. Section 4 explores the bar dependence on colors of host active galaxies with respect to both group central distance and group virial masses and Sect. 5 analyses environmental properties. In Sect. 6, we analyze the black hole activity in barred AGN in high density environments, and in Sect. 7 we summarize our main conclusions. The adopted cosmology through this paper is $\Omega=0.3, \Omega_{\lambda}=0.7$, and $H_{0}=100 \mathrm{~km} \mathrm{~s}^{-1} \mathrm{Mpc}$.

\section{Selection of barred AGN galaxies in high density environments}

The analysis of this paper is based on the SDSS-DR7 (Abazajian et al. 2009) photometric and spectroscopic galaxy catalog; DR7 is the seventh data release corresponding to the survey SDSSII, and comprises 11663 sq. deg. of sky imaged in five band widths $(u, g, r, i$, and $z)$. It provides imaging data for $357 \mathrm{mil}-$ lion objects, as well as spectroscopy over $\simeq \pi$ steradians in the north Galactic cap and 250 sq. deg. in the south Galactic cap. The SDSS-DR7 main galaxy sample is essentially a magnitude limited spectroscopic sample with $r_{\text {lim }}<17.77$ covering a redshift range $0<z<0.25$, with a median redshift of 0.1 (Strauss et al. 2002). For this work, several physical properties of galaxies were derived from the published SDSS-DR7 data: stellar mass content, indicators of star-bursts, emission-line fluxes, concentration index, etc. (Brinchmann et al. 2004; Blanton et al. 2005), and were obtained from the fits files at the MPA/JHU ${ }^{1}$ and the NYU ${ }^{2}$ catalogs.

In our previous work (A13) we compiled a barred AGN catalog obtained from SDSS-DR7. The active galaxy sample was selected by applying the Kauffmann et al. (2003) criteria, employing a standard diagnostic diagram proposed by Baldwin et al. (1981, hereafter BPT), and by using the publicly available emission-line fluxes (for details see A13). By visual inspection of SDSS images we classified 6772 hosts of face-on $(b / a>0.4)$ AGN spiral galaxies brighter than $g-$ mag $<16.5$ and with redshifts $z<0.1$ as barred or unbarred. We found 1927 AGN hosted in barred galaxies, representing a fraction of $28.5 \%$ with respect to the full sample of AGN in spiral host galaxies, and this value agrees with the bar fraction found by visual inspection of optical galaxy samples in previous works (de Vaucouleurs et al. 1991; Nilson 1973; Marinova et al. 2009; Masters et al. 2011; Oh et al. 2012). The remaining 4638 face-on spiral galaxies are unbarred. The authors excluded 207 objects that did not match the bar classification completely.

To analyze the properties of barred AGN galaxies in high density environments in detail, in comparison with AGN without bars, we identified barred and unbarred active objects that reside in groups and clusters by cross-correlating the total barred and unbarred AGN samples with the SDSS-DR7 group catalog constructed by Zapata et al. (2009). These authors identified groups of galaxies using a friends-of-friends algorithm with the same parameters corresponding to the values found by Merchan \& Zandivarez (2005) to produce a reasonably complete sample $(95 \%)$ with low contamination $(<8 \%)$. The group catalog has 122962 galaxies within 12630 groups that have a minimum number of four members and $z<0.1$. Moreover, it provides several useful galaxy group properties used throughout the paper, such as the geometric group center coordinates, virial mass, richness, line-of-sight velocity dispersion, and virial radius.

As a result of this cross-correlation, we obtained a sample of 319 barred and 519 unbarred AGN galaxies in groups. These values represent a fraction of $16.6 \%$ with respect to the full sample of 1927 barred AGN galaxies, and $11.2 \%$ with respect to a total of 4638 AGN without bars. Table 1 lists the numbers

\footnotetext{
http://www.mpa-garching.mpg.de/SDSS/DR7/

http://sdss.physics.nyu.edu/vagc/
} 


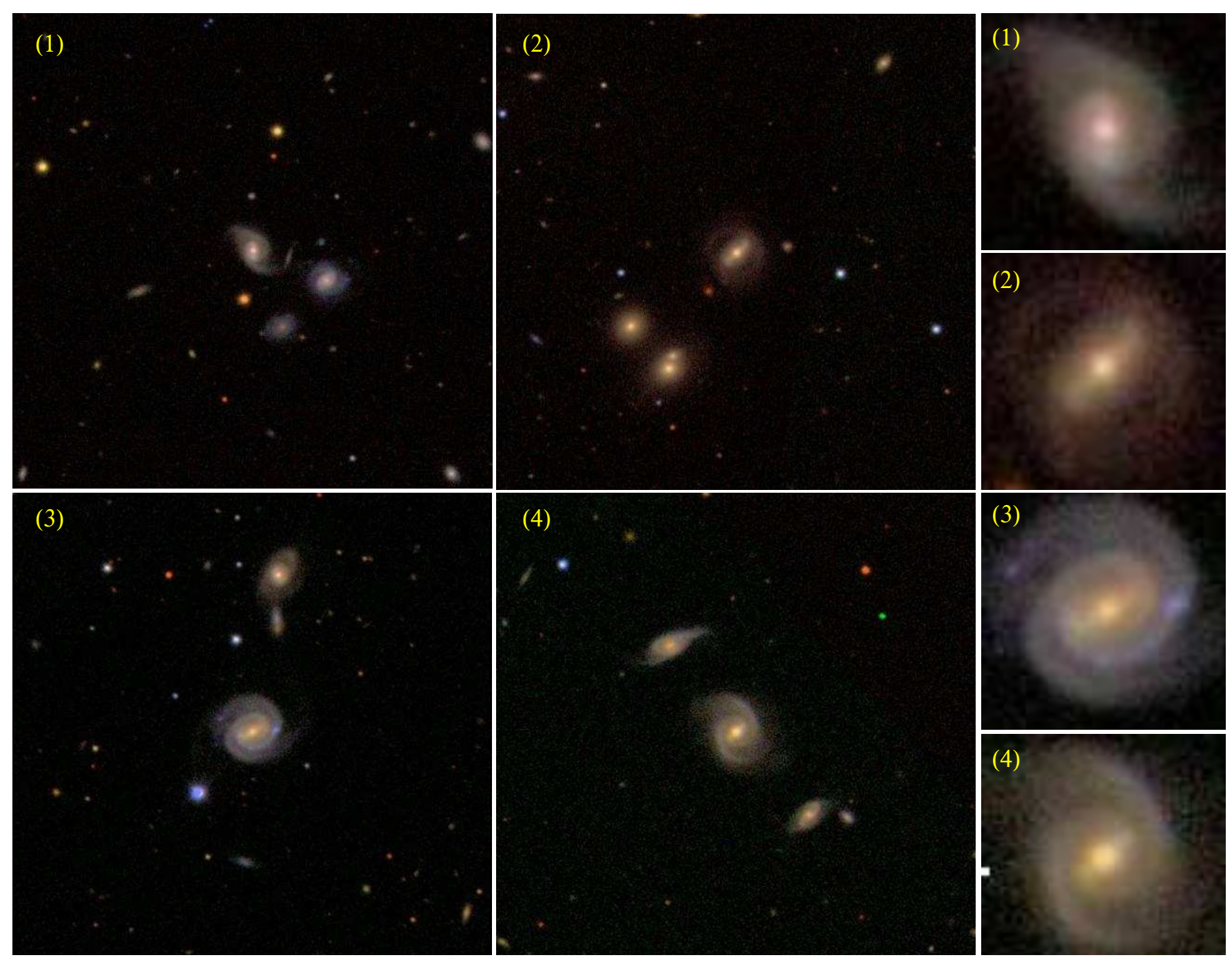

Fig. 1. Typical examples of barred active galaxies in high density environments. The images are $\approx 3.5 \times 3.5$. Right images show a zoom of the barred AGN galaxies.

Table 1. Results of classification.

\begin{tabular}{ccc}
\hline \hline Classification & Number of galaxies & Percentages \\
\hline Barred & 1927 & $28.5 \%$ \\
Unclear barred & 207 & $3.0 \%$ \\
Unbarred & 4638 & $68.5 \%$ \\
\hline Total & 6772 & $100.0 \%$ \\
\hline Barred in groups & 319 & $38.2 \%$ \\
Unbarred in groups & 519 & $61.8 \%$ \\
\hline Total & 838 & $100.0 \%$ \\
\hline
\end{tabular}

and percentages of the different AGN galaxy samples used in this work, and here we observed that the fraction of barred AGN galaxies in groups $(\approx 38 \%)$ is higher than those in the total barred AGN sample $(\approx 28 \%)$. This finding shows a higher bar fraction in groups and clusters of galaxies, indicating that AGN spiral galaxies in groups are more likely to be barred than those in the field, in agreement with previous work by Skibba et al. (2012) and Eskridge et al. (2000). This suggests that bars are stimulated in accordance with the local density environment and that the physical processes such as ram pressure stripping, starvation or strangulation, harassment and interactions in groups and clusters seem to be related to the bar phenomenon. Skibba et al. (2012) found that bars are formed by secular evolution and that this process depends on the local environment of the host galaxies, suggesting that there is a dichotomy between internal secular mechanisms and external environment. Recently, Kormendy \& Bender (2012) show that harassment may influence secular evolution of the bars by the cumulative effect of several rapid encounters with other cluster members. In addition, starvation or strangulation, which are gas stripping processes of the hot diffuse component of satellite galaxies, influences the star formation on longer timescales (Larson 1980) and could also stimulate the growing bars (Berentzen et al. 2007; Masters et al. 2012). Figure 1 shows images of typical examples of barred AGN host galaxies in high density environments selected from our sample.

In addition, we calculated the bar fraction as a function of colors and stellar masses of the host galaxies in our sample (see Table 2). We can observe that the bar fraction increases towards redder and more massive AGN host galaxies, in accordance with previous work (Masters et al. 2011; Oh et al. 2012).

\subsection{Control sample}

Some studies of the effect of bars from observational analysis show contradictory conclusions. In several cases, authors have attempted to isolate the effects of bars by comparing barred 
Table 2. Percentages of barred and unbarred active galaxies with different color and stellar mass ranges.

\begin{tabular}{ccc}
\hline \hline Color ranges & Barred AGN & Unbarred AGN \\
$0.55<\left(M_{g}-M_{r}\right)<0.7$ & $26.6 \%$ & $73.4 \%$ \\
$0.7<\left(M_{g}-M_{r}\right)<0.85$ & $45.4 \%$ & $54.6 \%$ \\
$0.85<\left(M_{g}-M_{r}\right)<1.0$ & $42.5 \%$ & $57.5 \%$ \\
\hline Stellar mass ranges & & \\
$10.2<\log \left(M_{*} / M_{\odot}\right)<10.6$ & $31.2 \%$ & $68.8 \%$ \\
$10.6<\log \left(M_{*} / M_{\odot}\right)<11.0$ & $40.0 \%$ & $60.0 \%$ \\
$11.0<\log \left(M_{*} / M_{\odot}\right)<11.4$ & $40.5 \%$ & $59.5 \%$ \\
\hline
\end{tabular}

galaxies with unbarred ones. However, different authors have proposed different ways to build comparison samples, and so the discrepancy in the results could be mainly due to a biased selection of these control samples. By using SDSS mock galaxy catalogs built from the Millennium Simulation, Pérez et al. (2009) showed that a suitable control sample for pair galaxies should be selected that at least match, redshift, morphology, stellar masses, and local density environments. This criteria is also applicable to the building of control samples of barred galaxies with the purpose of analysing different properties with respect to unbarred ones.

Our aim is to focus on the effects of bars on the active nuclei galaxies that reside in high density environments. Therefore, we constructed a reliable control sample of unbarred AGN host galaxies that share similar environmental conditions in order to compare these with the barred host results. Using a Monte Carlo algorithm we selected unbarred active galaxies in groups with similar redshift and stellar mass distributions (see panels $a$ and $b$ in Fig. 2). We have also considered unbarred AGNs in the control sample with similar concentration indices ${ }^{3}, C$, as for the barred AGN host sample, with the purpose of obtaining a similar bulgeto-disk ratio (see panel c). Thus, the differences in the results will be driven by the presence of bars and not by the difference in the morphology of the galaxies. In addition, we have take into account active galaxies with similar bulge prominences in both AGN samples, selecting control galaxies with similar distributions of the fracdeV parameter to the barred AGN host sample (panel d in Fig. 2). This parameter is a good indicator of bulge sizes in disk galaxies (Bernardi et al. 2010; Kuehn 2005; Masters et al. 2011; Skibba et al. 2012). The methodology followed to obtain the control sample of unbarred active galaxies in this work ensures that it will have the same selection effects as the barred AGN host catalog. It can then be used to estimate the real difference between active galaxies with and without bars in groups and clusters, unveiling the effect of bars on the nuclear activity and the role played by high density environments.

\section{Analysis of the relative position in groups}

The study of the bar fraction in the extremely dense regions of the cluster core with respect to the lower density environments of the outer regions of the galaxy clusters can provide important clues to understanding the relative effects of internal and external physical mechanism on the evolution of the barred disk galaxies. We may ask whether barred AGN galaxies have a particular location in groups and clusters with respect to the control sample. Therefore, we analyze the distribution of projected radial distance with respect to group centers, $d_{\mathrm{CG}}$, for barred AGN galaxies and AGN without bars in the control sample. In order to calculate reliable group centers, we have only

\footnotetext{
$3 C=r 90 / r 50$ is the ratio of Petrosian $90 \%-50 \%$-band light radii.
}
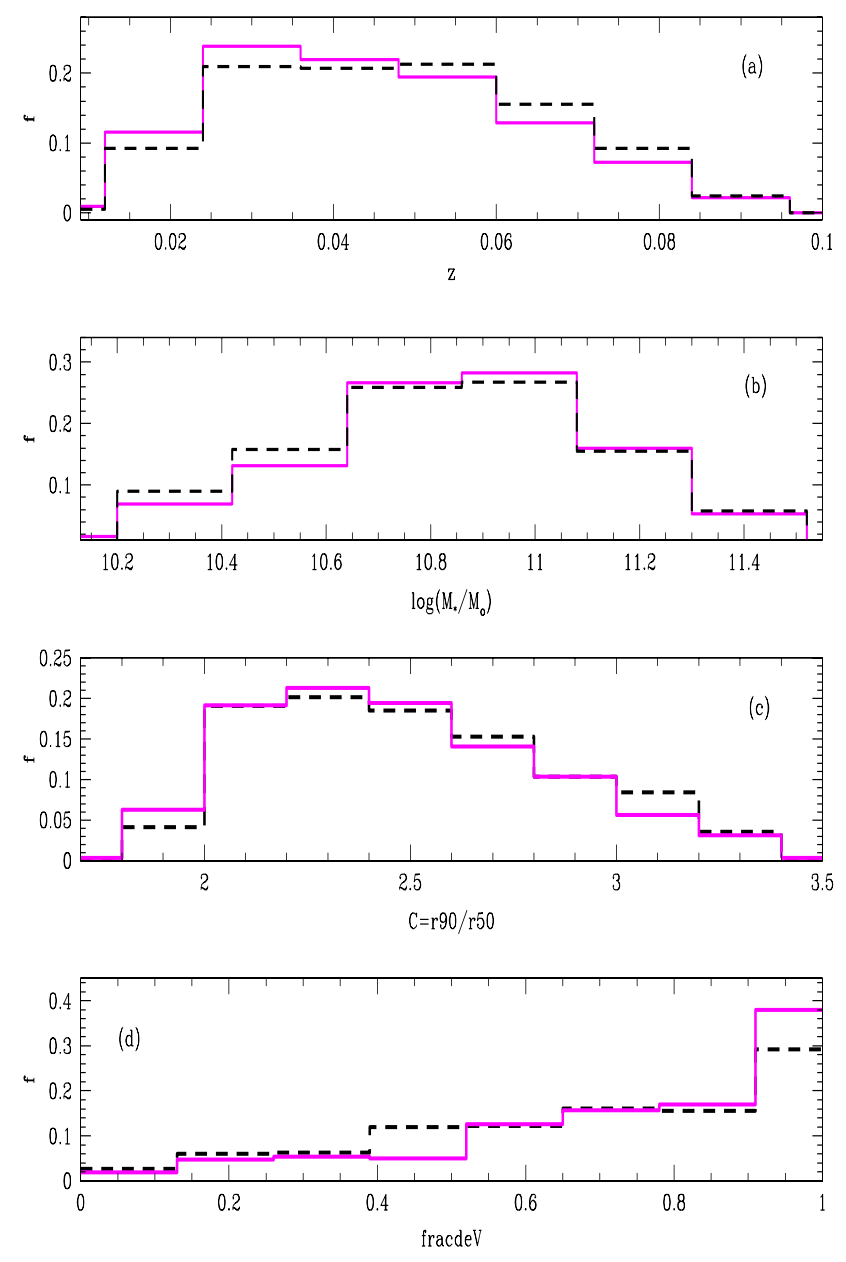

Fig. 2. Distributions of redshift $(z)$, stellar mass $\left(\log \left(M_{*}\right) / M_{\odot}\right)$, concentration index $(C)$, and bulge size indicator ( fracdeV) (panels a), b), c), and d)), for barred AGN host galaxies (solid lines) and active galaxies without bars in the control sample (dashed lines).

considered groups with a minimum number of seven members, thereby avoiding large uncertainties in this determination. Both the virial masses and virial radius distributions of the restricted sample are similar to the total group sample. The resulting distributions are shown in Fig. 3. It can be clearly seen that barred AGN galaxies are more concentrated towards the group center than the unbarred AGN in the control sample. We also calculated the normalized group-centric distance, $d_{\mathrm{CG}} / R_{\text {Virial }}$, finding the same tendencies (see the inset). In addition, we find that the fraction of barred AGN galaxies nearest the group-centric distance, $d_{\mathrm{CG}}<300 \mathrm{kpc} h^{-1}$, is higher $(\approx 49 \%)$ with respect to those in the unbarred AGN sample $(\approx 38 \%)$.

The pioneer work of Thompson (1981) also showed that the bar fraction is significantly larger in the core of the Coma cluster than in the outer region.

More recently, Marinova et al. (2012), using high resolution images from the Hubble Space Telescope ACS Treasury survey of the Coma cluster at $z \approx 0.02$, studied bars in the dense core of this cluster. They found a hint of an increase in the mean bar fraction toward the core of the Coma. In addition, the study of the bars in lenticular galaxies (S0) in the rich Coma cluster showed that there is a radial increase in the bar fraction towards the cluster core with respect to the low density outer regions (Lansbury et al. 2014). Andersen (1996) found that the radial distribution of the barred spirals in the Virgo cluster is clearly different than that 


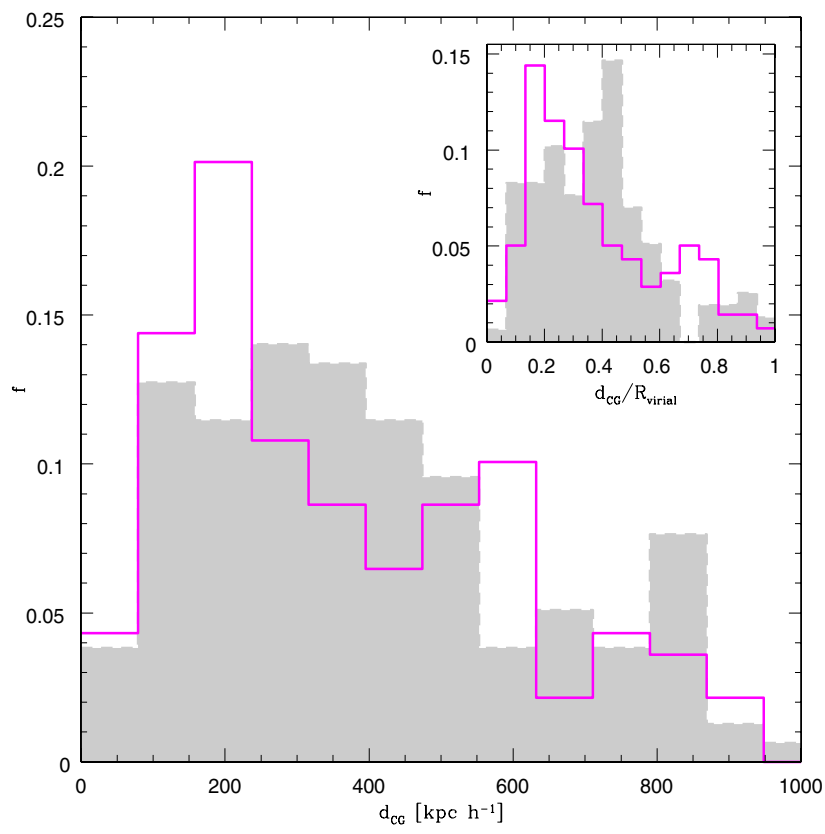

Fig. 3. Distributions of group-centric galaxy distances, $d_{\mathrm{CG}}$, for barred AGN (solid lines) and unbarred AGN (full surface) in the control sample. The inset shows to the distribution of the normalized group-centric distance, $d_{\mathrm{CG}} / R_{\text {Virial }}$, in the same samples.

of the spirals without bars, showing that barred galaxies are more centrally condensed than the unbarred ones. Moreover, observational studies in several galaxy clusters also found an increase in the bar fraction in the dense core of the clusters (Andersen 1996; Barazza et al. 2009; Marinova et al. 2012).

From numerical studies, Byrd \& Valtonen (1990) argued that the observed tendency for disk galaxies in the center of rich clusters to be barred is due to the strong tidal field. This can transform unbarred spiral galaxies into barred ones. In this context, the tidal field has a constant direction and is symmetric about the spiral galaxy, as would be the case for cluster members approaching in an eccentric orbit towards the cluster center. This symmetric perturbation can produce distortions in the galaxy disk and then form strong arms and bars. In addition, other tidal effects of the cluster field have been studied, e.g., tidal stripping (Merritt 1984) and tidal disruption (Miller 1986). These authors also found that galaxies in the core of the groups and clusters can survive over the long term without being destroyed. Our results confirm these previous findings for statistically reliable observational data.

\section{Color in host AGN galaxies}

Galaxy colors, as is well known, are correlated with star formation, age, gas content, and environment, all of which may be related to the presence of disk instabilities such as bars, and with the central nuclear activity. With the aim of exploring the colors of the barred active nuclei galaxies and the effects of denser regions, in this section we analyze colors in both samples of AGN with and without bars that inhabit groups and clusters.

In Fig. 4 we show the $(M u-M r)$ and $(M g-M r)$ color distributions of barred and unbarred active galaxies residing in groups and clusters. It can be clearly seen that there is a significant excess of barred AGN hosts with red colors. In Table 3 we quantify the excess of red color indexes $((M u-M r)>2.4$ and
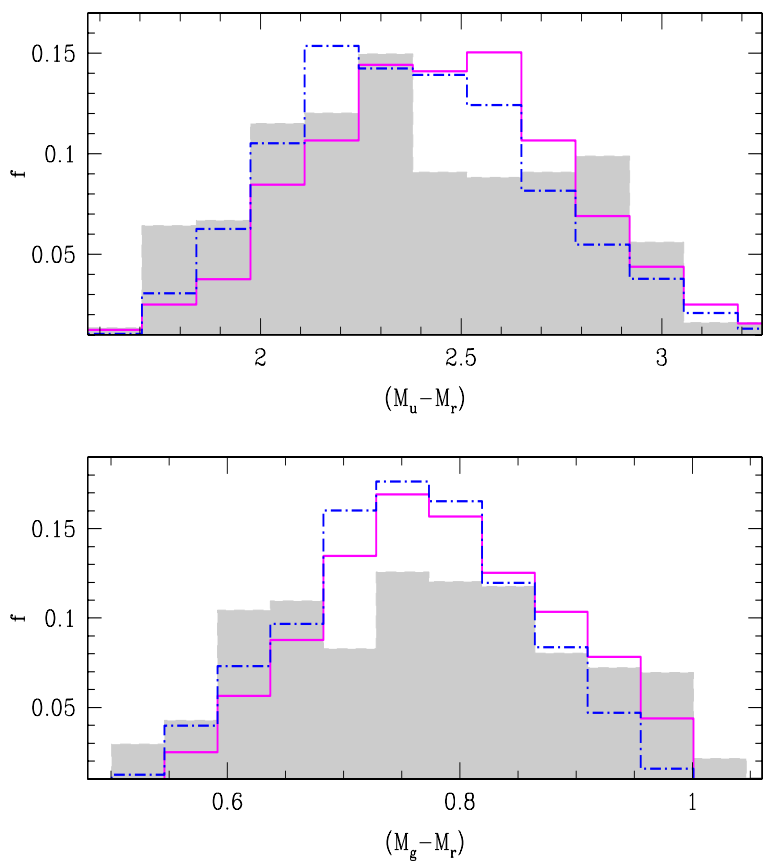

Fig. 4. Distribution of colors, $(M u-M r)$ and $(M g-M r)$ (upper and lower panels), for barred AGN host galaxies (solid lines) and AGN hosts without bars (shaded histograms) within groups and clusters. Dotdashed lines represent barred AGN hosts in the field.

Table 3. Percentages of barred and unbarred AGN host galaxies in groups with red colors.

\begin{tabular}{ccc}
\hline \hline Restrictions & \% of barred & \% of unbarred \\
\hline$(M u-M r)>2.4$ & $56.2 \%$ & $40.3 \%$ \\
$(M g-M r)>0.65$ & $89.6 \%$ & $75.7 \%$ \\
\hline
\end{tabular}

$(M g-M r)>0.65)$ of barred AGN hosts with respect to the unbarred AGN in the control sample.

Several previous studies displayed an excess of barred galaxies with redder colors from different samples. Masters et al. (2011) found a high fraction of bars in the passive red spiral galaxies for a sample obtained from the Galaxy Zoo catalog. In addition, $\mathrm{Oh}$ et al. (2012) showed that a significant number of barred galaxies are redder than their counterparts of unbarred spiral galaxies. Furthermore, in our previous work (A13) we found an excess of red colors in a sample of isolated spiral barred AGN with respect to unbarred active galaxies in a suitable control sample. The results obtained here from barred AGN hosts in high density environments are consistent with those of barred galaxies in low density regions.

In addition, we analyze the colors of barred AGN hosts in denser regions in comparison with those of active galaxies with bars in the field. For this purpose we used isolated barred AGN hosts obtained from our previous work (see A13 for details). This sample has similar redshift, stellar mass, concentration index, and bulge size indicator distributions to the sample of barred active galaxies in high density environments. From Fig. 4 we can see that there is an excess of barred AGN hosts with redder colors in groups and clusters with respect to isolated barred active galaxies. Furthermore, we calculated the excess of red color indexes for the isolated sample, $(M u-M r)>2.4$ and $(M g-M r)>0.65$, finding $46.5 \%$ and $84.8 \%$, respectively. This result also shows that isolated barred AGN hosts are redder than 


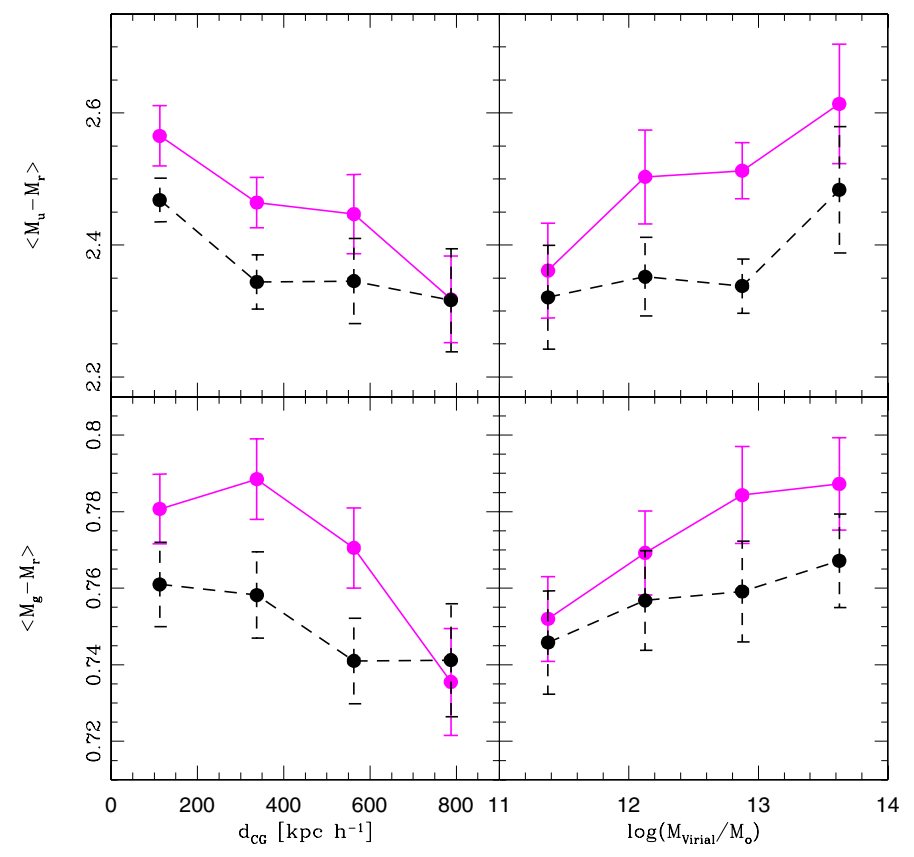

Fig. 5. Mean $\langle M u-M r\rangle$ and $\langle M g-M r\rangle$ galaxy colors (upper and lower panels) as a function of the group-centric galaxy distances, $d_{\mathrm{CG}}$ (left panels), and virial group mass, $\log \left(M_{\mathrm{V} \text { irial }} / M_{\odot}\right)$ (right panels), for barred and unbarred AGN host galaxies (solid and dashed lines).

unbarred active galaxies in groups, suggesting that bars determine changes in the host colors.

Moreover, to help us to understand the behavior of the colors in barred AGN hosts within high density environments, we measured the mean color values with respect to the group-centric distance. In the left panels of Fig. 5, we show the mean $\langle M u-M r\rangle$ (upper panel) and $\langle M g-M r\rangle$ (lower panel) colors as a function of group-centric distance for both samples of barred and unbarred active galaxies. As is well known, the central regions of the groups and clusters of galaxies are mainly populated by objects redder than those in the outer regions. It can also be seen that the number of active galaxies with redder colors increases toward the group centers, and this tendency is more significant in barred AGN with respect to those in the control active galaxies without bars.

Figure 5 (right panels) shows the mean colors for barred AGN (solid lines) as a function of the host group virial mass. We also show the results for the control sample (dashed lines). As can be seen, the number of redder AGN hosts increases towards higher group virial masses. In addition, we can see that barred AGN have systematically redder colors than active galaxies without bars in the control sample.

Redder colors found in barred active galaxies, with respect to unbarred counterparts, suggest that bar perturbations have a considerable effect on colors of AGN hosts.

\section{Local environmental properties}

In this section, we analysed the host group properties. For this purpose we explore the virial masses and the group colors in both host groups of barred and unbarred active galaxies. We calculated the group color, $\left(M_{u}-M_{r}\right)_{\text {Group }}$, which is measured by summing the luminosities of the four brightest galaxy members in the $r$ and $u$ bands in each group. Different authors (Eke et al. 2004; Padilla et al. 2004) found that this measure is a good parameter with which to quantify the global group luminosity.
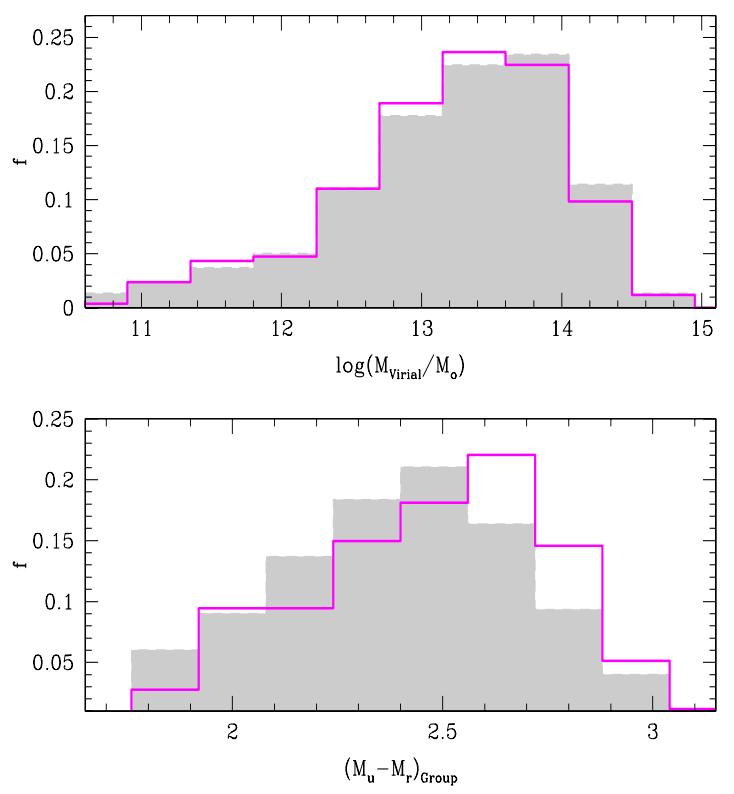

Fig. 6. Distributions of group virial masses, $\log \left(M_{\text {Virial }} / M_{\odot}\right)$ (upper panel), and group colors, $\left(M_{u}-M_{r}\right)_{\text {Group }}$ (lower panel), for host groups of barred and unbarred AGN galaxies (solid lines and full surface, respectively).

The results are plotted in Fig. 6 where we show the distributions of the virial masses, $\log \left(M_{\mathrm{Virial}} / M_{\odot}\right)$ (upper panel), and the group colors, $\left(M_{u}-M_{r}\right)_{\text {Group }}$ (lower panel), of the groups hosting, for barred AGN and their unbarred counterparts. We find that both host groups of the barred and unbarred AGN show similar virial mass distributions. Interestingly, the group color distributions of both samples show significant differences. The host groups of the corresponding barred active galaxies show a larger fraction of red colors with respect to the host groups of the unbarred galaxies in the control sample. We calculate the fraction of active galaxies with and without bars that reside in red groups, defined as $\left(M_{u}-M_{r}\right)_{\text {Group }}>2.4$ and find a percentage of about $62.2 \%$ and $51.8 \%$, respectively.

Figure 7 shows color-magnitude diagrams for host groups of barred active galaxies and unbarred AGN in the control sample. In addition, the analysis was performed for two ranges of group virial masses: $M_{\text {Virial }}<10^{13.5} M_{\odot}$ and $M_{\text {Virial }}>10^{13.5} M_{\odot}$ (middle and lower panels, respectively). As can be observed, for the same $\left(M_{r}\right)_{\text {Group }}$, the host group colors of barred active galaxies show distributions spreading toward red populations with respect to the host group colors of the unbarred AGN objects. In addition, this trend is more significant in less massive groups. However, groups with $M_{\text {Virial }}>10^{13.5} M_{\odot}$ have a more similar color-magnitude distributions than that of host groups of active galaxies without bars.

\section{Nuclear activity in barred AGN galaxies}

With the aim of assessing the effect of bars on the nuclear activity and the role played by high density environments, in this section we analyzed the central activity in the samples of AGN galaxies with and without bars in groups and clusters. We focused on the dust-corrected luminosity of the [OIII] $\lambda 5007$ line, Lum[OIII], as a tracer of the AGN activity. The [OIII] line is the strongest narrow emission line in optically obscured AGN and has a low contamination from contributions of star formation lines in the host galaxy. Furthermore, the Lum[OIII] 

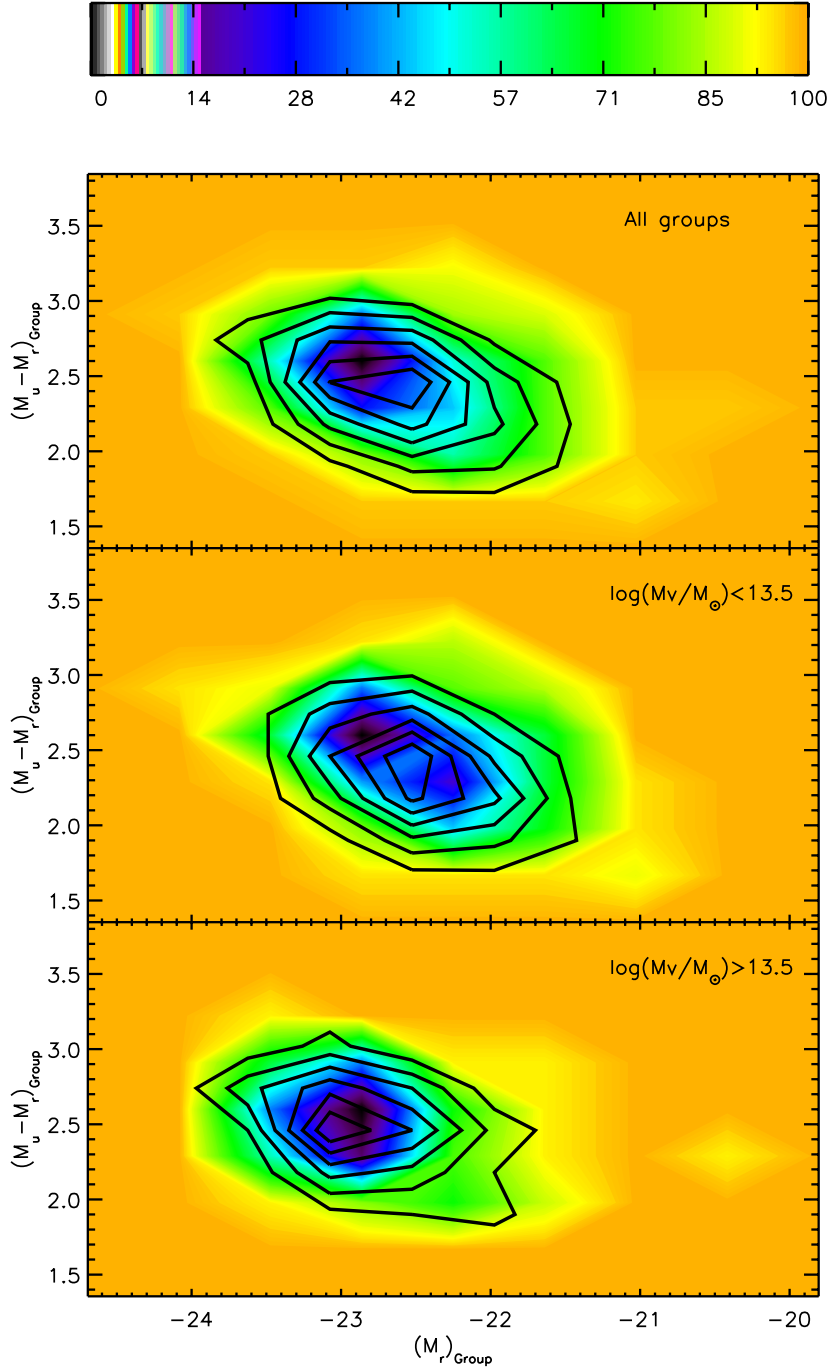

Fig. 7. Color-magnitude diagrams $\left(M_{u}-M_{r}\right)_{\text {Group }}$ versus $\left(M_{r}\right)_{\text {Group }}$. The density map shows the host groups of barred AGN (color scale corresponding to the cumulative percentage of objects, as shown in the key). For comparison, the black solid lines enclose $14 \%, 28 \%, 42 \%, 57 \%$, $71 \%$, and $85 \%$ of the host groups of unbarred AGN in the control sample. Middle and lower panels show host groups with different ranges of virial masses: $M_{\text {Virial }}<10^{13.5} M_{\odot}$ and $M_{\text {Virial }}>10^{13.5} M_{\odot}$, respectively.

estimator has been widely used by different authors in several works (Mulchaey et al. 1994; Kauffmann et al. 2003; Heckman et al. 2004, 2005; Brinchmann et al. 2004). From an inspection of the BPT diagrams, Kauffmann et al. (2003) found that high metallicity hosts show a low contamination from star formation in the Lum[OIII]. In our sample, most of the galaxies have stellar masses $M^{*}>10^{10} M_{\odot}$ (see panel b in Fig. 2), therefore their metallicities are expected to be high because of the mass-metallicity relation (Kauffmann et al. 2003; Tremonti et al. 2004), with low contamination of star formation lines.

In Fig. 8 we show the distributions of Lum[OIII] for AGN with and without bars within groups and clusters. We also plot isolated barred active galaxies residing in the field. It is interesting that in high density regions barred AGN show a slight trend toward higher values of Lum[OIII] than AGN without bars in the control sample. Moreover, isolated active galaxies with bars show an excess of higher nuclear activity values with respect to those of barred AGN within groups and clusters. Following A13, we divide the sample by using the Lum[OIII] to

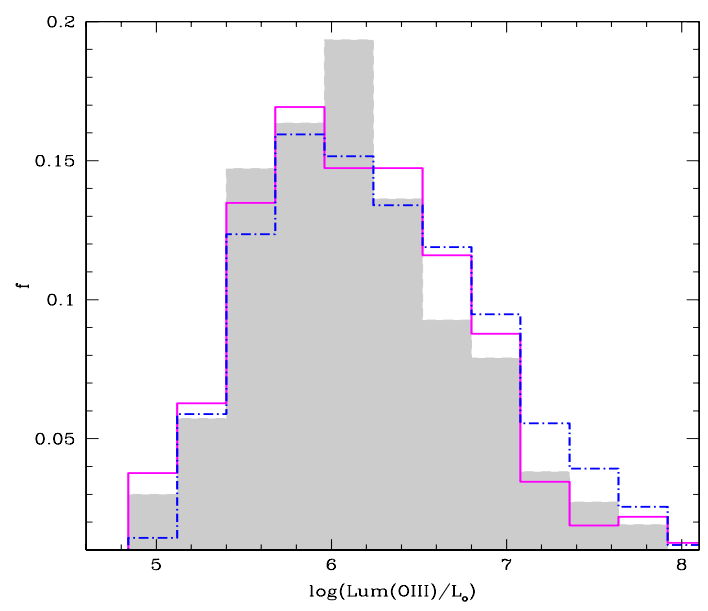

Fig. 8. Distributions of $\log \left(\operatorname{Lum}[\mathrm{OIII}] / L_{\odot}\right)$ for barred AGN (solid line) and unbarred active galaxies (full surfaces) in high density environments. Dot-dashed line represents isolated barred AGN galaxies.

study trends with luminosity. Then, we consider the luminosity Lum $[\mathrm{OIII}]=10^{6.4} L_{\odot}$ as a limit between the lower-luminosity and the higher-luminosity subsamples. Thus, we estimate that the $43.4 \%, 35.7 \%$, and $31.5 \%$ of isolated barred active objects, barred, and unbarred AGN hosts in denser regions, respectively, belong to the higher-luminosity subsample, because they are the strongest AGN. These findings indicate that the central nuclear activity in barred AGN galaxies is more efficient in lower density regions than in higher density environments.

Figure 9 (upper and middle panels) shows the nuclear activity distributions, $\log \left(\operatorname{Lum}[\mathrm{OIII}] / L_{\odot}\right)$, of barred active galaxies compared with the unbarred AGN host counterparts. The analysis was performed for the different ranges of group-centric distances $\left(d_{\mathrm{CG}}<500 \mathrm{kpc} h^{-1}\right.$ and $\left.d_{\mathrm{CG}}>500 \mathrm{kpc} h^{-1}\right)$, with the purpose of studying the efficiency of nuclear activity in AGN galaxies with and without bars located in the cluster cores and in the regions far from the group centers. Different authors (Sarazin 1980; Kent \& Gunn 1982) found that the values of the cluster core radius are typically between $300 \mathrm{kpc}$ and $600 \mathrm{kpc}$. Dressler (1980) also obtained similar values for the sample of 55 rich clusters. Moreover, the X-ray emitting gas gives a suitable proxy to the mass distribution in the clusters, which is an important parameter for obtaining the core radius. In this context, several X-ray observations (Forman \& Jones 1982; Patel et al. 2000; Haarsma et al. 2010) usually imply core radii of $\approx 500 \mathrm{kpc}$. We found that towards the group center, the Lum[OIII] distributions of barred AGN hosts are similar to those of AGN without bars in the control sample. Interestingly, towards regions farthest from the group center, the activity distributions of barred and unbarred AGN hosts exhibit significant differences, with barred objects having a higher fraction of powerful AGN. We have also computed the mean $\left\langle\log \left(\operatorname{Lum}(\mathrm{OIII}) / L_{\odot}\right)\right\rangle$ values for different bins of group-centric distance, $d_{\mathrm{GC}}$, for both $\mathrm{AGN}$ samples. The results from the lower panel in Fig. 9 show clearly that, in barred AGN for larger group-centric distance, the Lum[OIII] values strongly increases, while in active galaxies without bars in the control sample remains approximately constant with a slight decline. It could be interpreted in terms of the greater ability of bars to fuel the central black holes in host group member galaxies located far from the group center.

With the aim of understanding the behavior of the nuclear activity in AGN galaxies with and without bars as a function of host group colors, in Fig. 10 we show the nuclear 

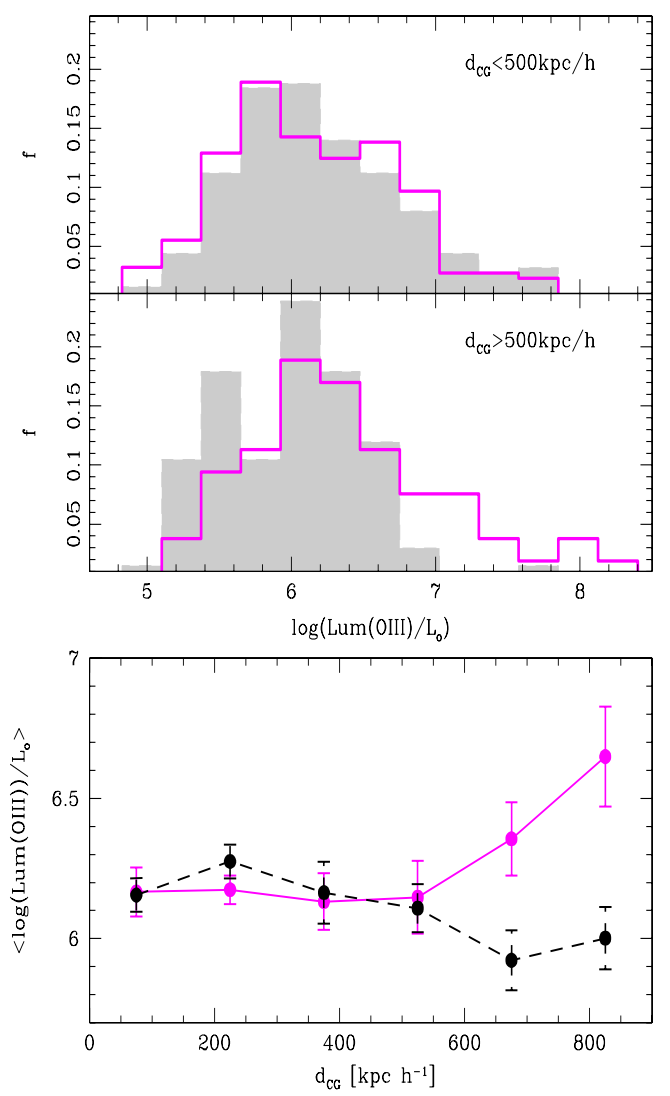

Fig. 9. Distributions of $\log \left(\operatorname{Lum}[\mathrm{OIII}] / L_{\odot}\right)$ for barred AGN (solid lines) and unbarred active galaxies (full surfaces) with different group-centric distances: $d_{\mathrm{CG}}<500 \mathrm{kpc} h^{-1}$ and $d_{\mathrm{CG}}>500 \mathrm{kpc} h^{-1}$ (upper and middle panels, respectively). Lower panel shows mean $\left\langle\log \left(\operatorname{Lum}(\mathrm{OIII}) / L_{\odot}\right)\right\rangle$ as a function of group-centric distance, $d_{\mathrm{CG}}$, for AGN galaxies with and without bars in groups (solid and dashed lines, respectively).

activity distributions for different ranges of group colors: $\left(M_{u}-\right.$ $\left.M_{r}\right)_{\text {Groups }}>2.4$ and $\left(M_{u}-M_{r}\right)_{\text {Groups }}<2.4$ (upper and middle panels, respectively). It can be clearly seen that barred AGN show a trend toward higher values of Lum[OIII] than AGN without bars in the control sample. This tendency is more significant in active galaxies that inhabit bluer groups. Moreover, in Table 4 we quantify the percentage of barred and unbarred active galaxies in denser regions with $\operatorname{Lum}[\mathrm{OIII}]=10^{6.4} L_{\odot}$, for different ranges of group-centric distance and host group colors.

In addition, in Fig. 10 (lower panel) we present the mean $\left\langle\log \left(\operatorname{Lum}[\mathrm{OIII}] / L_{\odot}\right)\right\rangle$ values as a function of the global group colors. This relation was calculated for the corresponding host groups of the barred AGN and for active galaxies without bars in the control sample. The results clearly show that generally in bluer groups, active galaxies show higher Lum[OIII] values. We can also see that barred AGN objects systematically show higher nuclear activity, irrespective of the group colors. These findings show that the higher-luminosity subsample AGN reside preferentially in bluer groups, which could provide suitable conditions for the central black hole feeding. In this context, the most efficient nuclear activity in barred AGN galaxies with respect to their unbarred counterparts reflect that bars have an important role in helping the gas infall towards the central regions in active galaxies. Considering that the group colors, $\left(M_{u}-M_{r}\right)$, given by the four brightest members is a representative parameter of the global color of the groups, this result is in agreement with that found by several authors. Coldwell et al. (2009) show that the power of the AGN activity is strongly dependent
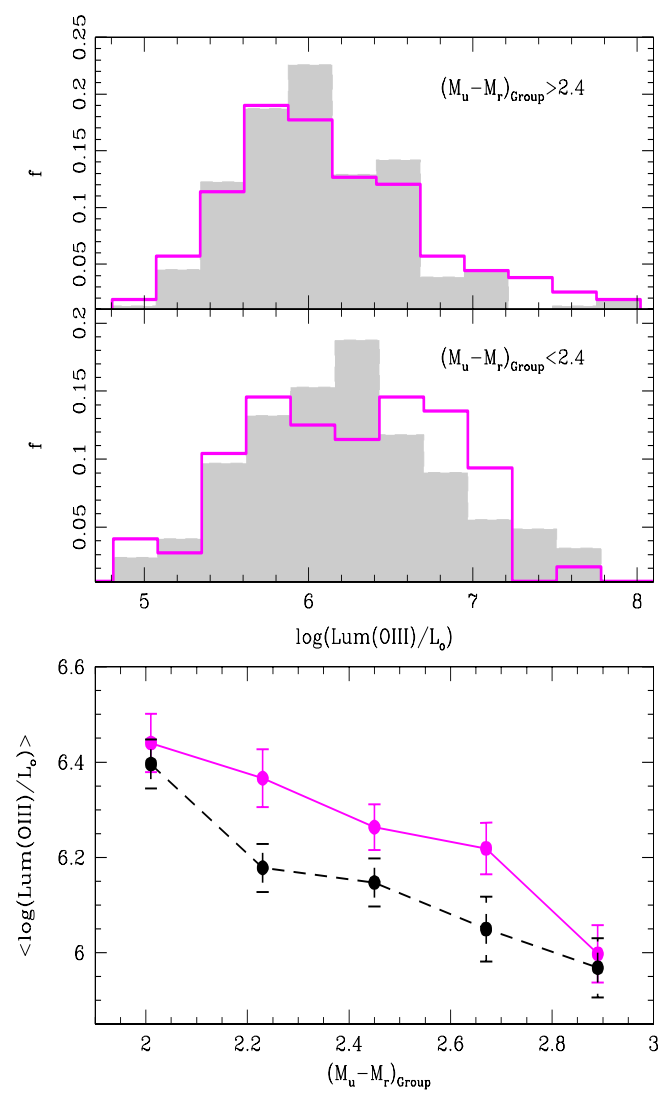

Fig. 10. Distributions of $\log \left(\operatorname{Lum}[\mathrm{OIII}] / L_{\odot}\right)$ for barred AGN (solid lines) and unbarred active galaxies (full surfaces) with different host group color ranges: $\left(M_{u}-M_{r}\right)_{\text {Groups }}>2.4$ and $\left(M_{u}-M_{r}\right)_{\text {Groups }}<2.4$ (upper and middle panels, respectively). Lower panel shows mean $\log \left(\operatorname{Lum}(\mathrm{OIII}) / L_{\odot}\right)$ as a function of the host group colors for AGN galaxies with and without bars in groups (solid and dashed lines, respectively).

Table 4. Percentages of barred and unbarred active galaxies with Lum $[\mathrm{OIII}]>10^{6.4} L_{\odot}$ in different ranges of group-centric distances and host group colors.

\begin{tabular}{ccc}
\hline \hline Ranges & \% of barred AGN & $\%$ of unbarred AGN \\
\hline$d_{\mathrm{CG}}<500 \mathrm{kpc} h^{-1}$ & $34.1 \%$ & $34.4 \%$ \\
$d_{\mathrm{CG}}>500 \mathrm{kpc} h^{-1}$ & $41.5 \%$ & $20.8 \%$ \\
\hline$\left(M_{u}-M_{r}\right)_{\text {Groups }}>2.4$ & $31.1 \%$ & $26.3 \%$ \\
$\left(M_{u}-M_{r}\right)_{\text {Groups }}<2.4$ & $43.8 \%$ & $34.2 \%$ \\
\hline
\end{tabular}

on the environment, finding that the AGN with higher values of $\left\langle\log \left(\operatorname{Lum}[\mathrm{OIII}] / L_{\odot}\right)\right\rangle$ are located in regions populated by bluer galaxies. Similar results were found by Padilla et al. (2010); Coldwell et al. (2014) suggesting that AGN very close to the high density regions need more available gas to effectively feed the central black hole. This is reflected in the higher probability of finding AGN in regions populated by bluer galaxies.

\section{Summary and conclusions}

We have performed a statistical analysis of local environmental properties, host characteristics, and nuclear activity of the AGN spiral galaxies with and without bars within denser regions. For this purpose, we have identified barred and unbarred objects that inhabit groups and clusters of galaxies, by crosscorrelating the total barred and unbarred AGN catalogs obtained 
from our previous work (see Alonso et al. 2013, for details) with the SDSS-DR7 group catalog constructed by Zapata et al. (2009). To obtain an appropriate quantification of the effects of bars on the active nuclei and the role played by high density environments, we constructed a suitable control sample of unbarred AGN galaxies within groups and clusters, with the same redshift, stellar mass, concentration index, and bulge size parameter distributions.

We now summarize the principal results of our analysis and its main conclusions.

(i) We found that the fraction of barred AGN galaxies in groups $(\approx 38 \%)$ is higher than those in the total barred AGN sample $(\approx 28 \%)$. This result shows that AGN spiral galaxies in groups are more likely to be barred than those in the field, indicating that galactic bars are stimulated in accordance with the local density environment, and also that the physical mechanisms produced within groups and clusters of galaxies (ram pressure, strangulation, harassment and interactions) seem to be related to the bar phenomenon (Kormendy \& Bender 2012; Berentzen et al. 2007; Masters et al. 2012). In addition, we observed that the bar fraction increases towards redder and more massive AGN host galaxies.

(ii) We have found that barred AGN galaxies are more concentrated towards the group centers than the other unbarred AGN galaxy members. We quantified this trend and we found that nearest the group-centric distance, $d_{\mathrm{CG}}<$ $300 \mathrm{kpc} h^{-1}$, the fraction of barred AGN galaxies is higher $(\approx 49 \%)$ with respect to those in the unbarred AGN sample $(\approx 38 \%)$. This implies that barred phenomenon are more likely to occur in the dense core of the groups and clusters, where the strong tidal field can transform unbarred spiral galaxies into barred ones (Byrd \& Valtonen 1990).

(iii) We examined the color distributions of barred and unbarred AGN host galaxies within groups and clusters, and we found that barred AGN hosts have a clear excess of redder colors than galaxies in the control sample. In addition, we analyzed the colors of AGN with bars in the field, finding that isolated barred AGN hosts are redder than active galaxies without bars in groups. This result suggests that bar perturbations can significantly affect galaxy colors in the hosts of AGN.

(iv) We also studied the mean colors in barred AGN galaxies and in the control sample as a function of the groupcentric distance and group virial masses. We found that the number of red galaxies increases toward the group center as expected, and barred galaxies have a higher fraction of redder colors than active galaxies without bars within groups/clusters. We also show that redder galaxies increases towards higher group virial masses and, in this context, barred AGN galaxies have systematically redder colors than active galaxies without bars in the control sample, for all virial mass ranges.

(v) We explored the host group properties of the AGN galaxies with and without bars, and we found that both host groups show similar virial masses. However, the group color distributions of both samples present significant differences; namely, the host groups of the barred AGN exhibit a larger fraction of red colors, with respect to the host groups of the corresponding unbarred active galaxies in the control sample. We also examined the color-magnitude relation for both host groups and we found that at the same $\left(M_{r}\right)_{\text {Group }}$, the host group colors of barred active galaxies display distributions spreading toward red populations with respect to the host groups of the unbarred AGN objects. This trend is more significant in less massive groups than in groups with $M_{\text {Virial }}>$ $10^{13.5} M_{\odot}$.

(vi) We found that in high density regions, barred active galaxies show an excess of nuclear activity compared to galaxies without bars in the control sample. We have also analyzed barred active galaxies in the field, and we found that isolated AGN galaxies with bars show more efficient nuclear activity with respect to those of barred AGN within groups and clusters.

(vii) We also analyzed the relation between the central nuclear activity with the group-centric distances, and with the global group colors for barred active galaxies and unbarred AGN in the control sample. From this study, we conclude that, in particular, the nuclear activity has strongly increased in barred active galaxies located far of the group center, while the nuclear activity in AGN galaxies without bars remains approximately constant with the group-centric distance. In addition, we found that in bluer host groups active galaxies show a higher Lum[OIII] values for both AGN samples; however, barred active objects systematically show a higher nuclear activity, irrespective of the global group colors. These results suggest that the efficiency of bars in transporting material towards the more central regions of the AGN galaxies in high density environments has a significant dependence on the localization of objects within the group and/or cluster and on the host group colors.

Acknowledgements. This work was partially supported by the Consejo Nacional de Investigaciones Científicas y Técnicas and the Secretaría de Ciencia y Técnica de la Universidad Nacional de San Juan. Funding for the SDSS has been provided by the Alfred P. Sloan Foundation, the Participating Institutions, the National Science Foundation, the U.S. Department of Energy, the National Aeronautics and Space Administration, the Japanese Monbukagakusho, the Max Planck Society, and the Higher Education Funding Council for England. The SDSS Web Site is http://www.sdss.org/. The SDSS is managed by the Astrophysical Research Consortium for the Participating Institutions. The participating institutions are the American Museum of Natural History, Astrophysical Institute Potsdam, University of Basel, University of Cambridge, Case Western Reserve University, University of Chicago, Drexel University, Fermilab, the Institute for Advanced Study, the Japan Participation Group, Johns Hopkins University, the Joint Institute for Nuclear Astrophysics, the Kavli Institute for Particle Astrophysics and Cosmology, the Korean Scientist Group, the Chinese Academy of Sciences (LAMOST), Los Alamos National Laboratory, the Max-Planck-Institute for Astronomy (MPIA), the Max-Planck-Institute for Astrophysics (MPA), New Mexico State University, Ohio State University, University of Pittsburgh, University of Portsmouth, Princeton University, the United States Naval Observatory, and the University of Washington.

\section{References}

Abazajian, K. N., Adelman-McCarthy, J. K., Ageros M. A., et al. 2009, ApJS, 182,543

Alonso, S., Coldwell, G., \& Lambas, D. G. 2013, A\&A, 549, A141

Andersen, V. 1996, AJ, 111, 1805

Arsenault, R. 1989, A\&A, 1989, 217, 66

Athanassoula, E. 2003, MNRAS, 341, 1179

Baldwin, J. A., Phillips, M. M., \& Terlevich, R. 1981, PASP, 93, 5

Barazza, F. D., Jablonka, P., Desai, V., et al. 2009, A\&A, 497, 713

Berentzen, I., Shlosman, I., Martinez-Valpuesta, I., \& Heller C. H. 2007, ApJ, 666,189

Bernardi, M., Shankar, F., Hyde, J. B., et al. 2010, MNRAS, 404, 2087

Blanton, M. Rr, Eisenstein, D., Hogg, D. W., Schlegel, D. J., \& Brinkmann, J. 2005, ApJ, 629, 143

Brinchmann, J., Charlot, S., White, S. D. M., et al. 2004, MNRAS, 351, 1151

Byrd, G., \& Valtonen, M. 1990, ApJ, 350, 89

Carollo, C. M., Stiavelli, M., Seigar, M., de Zeeuw, P. T., \& Dejonghe, H. 2002, AJ, 123, 159

Coldwell, G. V., Lambas, D. G., Soechting, I. K., \& Gurovich, S. 2009, MNRAS, 399,88 
Coldwell, G., Gurovich, S., Díaz Tello, J., Soechting, I. K., \& Lambas, D. G. 2014, MNRAS, 437, 1199

Combes, F., \& Elmegreen, B. G. 1993, A\&A, 271, 391

Corsini, E. M., Debattista, V. P., \& Aguerri, J. A. L. 2003, ApJ, 599, 29

Debattista, V. P., \& Sellwood, J. A. 1998, ApJ, 493, 5

Devereux, N. A. ApJ, 1987, 323, 91

Dressler, A. 1980, ApJS, 42, 565

de Vaucouleurs, G., de Vaucouleurs, A., Corwin, H. G., Jr., et al. 1991, 3rd e

Reference Catalogue of Bright Galaxies (USA, New York: Springer)

Eke, V. R., Frenk, C. S., Baugh, C. M., et al. 2004, MNRAS, 355, 769

Elmegreen, D. M., Elmegreen, B. G., \& Bellin, A. D. 1990, ApJ, 364, 415

Emsellem, E., Greusard, D., Combes, F., et al. 2001, A\&A, 368, 52

Eskridge, P. B. 2000, AJ, 119, 536

Forman, W., \& Jones, C. 1982, ARA\&A, 20, 547

Haarsma, D. B., Leisman, L., Donahue, M., et al. 2010, ApJ, 713, 1037

Hawarden, T. G., Mountain, C. M., Leggett, S. K., \& Puxley, P. J. 1986, MNRAS, 221,41

Heckman, T. M. 1980, A\&A, 88, 365

Heckman, T. M., Kauffmann, G., Brinchmann, J., et al. 2004, ApJ, 613, 109

Heckman, T. M., Ptak, A., Hornschemeier, A., \& Kauffmann, G. 2005, ApJ, 634, 161

Ho, L. C., Filippenko, A. V., \& Sargent, W. L. W. 1997, ApJ, 487, 591

Huang, J. H., Gu, Q. S., Su, H. J., et al. 1996, A\&A, 313, 13

Hunt, L. K., Combes, F., Garcia-Burillo, S., et al. 2008, A\&A, 482, 133

Jogee, S., Scoville, N., \& Kenney, J. D. P. 2005, ApJ, 630, 837

Kauffmann, G., Heckman, T. M., Tremonti, C., et al. 2003, MNRAS, 346, 1055

Kent, S. M., \& Gunn, J. E. 1982, AJ, 87, 945

Kewley, L. J., Dopita, M. A., Sutherland, R. S., Heisler, C. A., \& Travena, J., 2001, ApJ, 556, 121

Kewley, L. J., Groves, B., Kauffmann, G., \& Heckman, T. M. 2006, MNRAS, 372,961

Knapen, J.H., Perez-Ramirez, D., \& Laine, S. 2002, MNRAS, 337, 808

Kormendy, J., \& Bender, R. 2012, ApJS, 198, 2

Kuehn, F., \& Ryden, B. S. 2005, ApJ, 634, 1032

Laine, S., Shlosman, I., Knapen, J. H., \& Peletier, R. F. 2002, ApJ, 567, 97

Lansbury, G. B., Lucey, J. R., \& Smith, R. J. 2013, MNRAS, 439, 1749
Larson, R. B. 1980, MNRAS, 190, 321

Lee, G., Woo, J., Lee, M. G., et al. 2012, ApJ, 750, 141

Lintott, C., Schawinski, K., Bamford, S., et al. 2011, MNRAS, 410, 166

Maciejewski, W., \& Sparke, L. S. 1997, ApJ, 484, 117

Malkan, M. A., Gorjian, V., \& Tam, R. 1998, ApJS, 117, 25

Marinova, I., Jogee, S., Heiderman, A., et al. 2009, ApJ, 698, 1639

Marinova, I., Jogee, S., Weinzirl, T., et al. 2012, ApJ, 746, 136

Martinet, L., \& Friedli, D. 1997, A\&A, 323, 363

Martínez, H. J., \& Muriel H. 2011, MNRAS, 418, 148

Masters, K. L., et al. 2010, MNRAS, 411, 2026

Masters, K. L., Nichol, R. C., Haynes, M. P., et al. 2012, MNRAS, 424, 2180

Mendez-Abreu, J., Sanchez-Janssen, R., \& Aguerri, J. A. L. 2010, ApJ, 711, L61

Merchan, M. E., \& Zandivarez, A. 2005, ApJ, 630, 739

Merritt, D. 1984, ApJ, 276, 26

Miller, H. R. 1986, MNRAS, 220, 713

Mulchaey, J. S., \& Regan, M. W. 1997, ApJ, 482, 135

Mulchaey, J. S., Koratkar, A., Ward, M. J., et al. 1994, ApJ, 436, 586

Nilson, P. 1973, Acta Universitatis Upsaliensis. Nova Acta Regiae Societatis

Scientiarum Upsaliensis - Uppsala Astronomiska Observatoriums Annaler, Uppsala: Astronomiska Observatorium

Oh, S., Oh, K., \& Yi, S. K. 2012, ApJS, 198, 40

Padilla, N. D., Baugh, C. M., Eke, V. R., et al. 2004, MNRAS, 352, 211

Padilla, N. D., Lambas, D. G., \& González, R. 2010, MNRAS, 409, 936

Patel, S. K., Joy, M., Carlstrom, J. E., et al. 2000, ApJ, 541, 37

Pérez, J., Tissera, P., \& Blaizot, J. 2009, MNRAS, 397, 748

Sarazin, C. L. 1980, ApJ, 236, 75

Shlosman, I., Frank, J., \& Begelman, M. C. 1989, Nature, 338, 45

Shlosman, I., Begelman, M. C., \& Frank, J. 1990, Nature, 345, 679

Skibba, R. A., Masters, K. L., Nichol, R. C., et al. 2012, MNRAS, 423, 1485

Strauss, M. A., Weinberg, D. H., Lupton, R. H., et al. 2002, AJ, 124, 1810

Thompson, L. A. 1981, ApJ, 244, 43

Tremonti, C., Heckman, T. M., Kauffmann, G., et al. 2004, ApJ, 613, 898

van den Bergh, S. 2002, AJ, 124, 782

Weinberg, M. D. 1985, MNRAS, 213, 451

Zapata, T., Pérez, J., Padilla, N., \& Tissera, P. 2009, MNRAS, 394, 2229 